\title{
Reference to kinds in L2 English
}

\author{
Y1lmaz Köylü*
}

\begin{abstract}
This study investigated the acquisition of kind referring noun phrase interpretation in L2 English by learners with Turkish, Arabic and Chinese L1 backgrounds. 37 advanced learners of English with Turkish (10), Arabic (10) and Chinese (10) L1 backgrounds, and 7 native English speakers were recruited. The tasks were a 48-item fill in the gaps task and a 64-item acceptability judgment task. The results indicated that: (a) native speakers, and L2 learners mostly produced bare plurals for count nouns and bare singulars for mass nouns for kind reference; (b) L2 learners of English transferred the morphosyntactic manifestation of kind reference from their L1s, substantiating the Full Transfer Full Access Hypothesis (Schwartz \& Sprouse, 1996); and (c) the similarity between the participants' L1s and L2 did not always lead them to produce correct noun forms and articles for kind reference, neither did such a similarity consistently help the learners in their acceptability judgments for kind reference.
\end{abstract}

Keywords. genericity; kind reference; second language acquisition; Turkish; Arabic; Chinese

1. Introduction. Generic statements and kind referring expressions are conveyed using different nominal morphosyntax, and articles crosslinguistically. These expressions clearly pose a learnability problem in second language acquisition of English as they are seldom taught explicitly, and neither are such expressions abundant in the input to guide learners to use correct nominal morphosyntax, and articles. Nevertheless, L2 learners seem to acquire the morphosyntactic manifestation of kind reference successfully despite the poverty of the stimulus as evidenced by Ionin \& Montrul (2010); Ionin et al. (2011), and Ionin et al. (2014). The objective of this study is twofold: (a) to shed light on the effect of the first language in acquiring kind reference in second language English (L2 English); and (b) to investigate the developmental trajectory that second language learners go through in acquiring such subtle meanings at the syntax/semantics interface in L2 English.

2. Genericity and kind reference. According to Krifka, Pelletier, Carlson, ter Meulen, Link, and Chierchia (1995), genericity can be construed in terms of two distinct phenomena. The first one is reference to a kind, illustrated below.

(1) a. The polar bear is dying out.

b. Polar bears are dying out.

c. Milk is nutritious.

$(1 \mathrm{a} / \mathrm{b} / \mathrm{c})$ express some generalization about a kind. To be more specific, $(1 \mathrm{a} / \mathrm{b})$ are statements regarding the polar bear kind in general rather than being a property of individual polar bears. Similarly, (1c) states a general property of the kind of liquid known as milk. The

\footnotetext{
* I would like to thank Indiana University, College of Arts and Sciences, and the Department of Second Language Studies for their generous travel funds to the $93^{\text {rd }}$ Annual Meeting of the LSA, where I presented this paper. Special thanks to the audience at LSA 93 for their insightful comments and suggestions. Y1lmaz Köylü, Indiana University (ykoylu@indiana.edu). 
ungrammaticality of the following sentence to refer to a kind illustrates that an indefinite singular cannot be kind referring in English.

(2) *A polar bear is dying out.

Since the predicate die out can only be true of a kind rather than an individual in the kind, (2) is ungrammatical. Based on the examples in (1), Krifka et al. (1995) argue that only definite singulars, bare plurals and mass nouns can be kind referring in English.

The second type of genericity is a generic sentence that reports a regularity that summarizes groups of particular episodes or facts (Carlson \& Pelletier, 1995), illustrated below.

(3) a. A cat meows.

b. The cat meows.

c. Cats meow.

Krifka et al. (1995) refer to sentences in (3) as characterizing sentences, or simply generic sentences since they express generalizations. Characterizing sentences can be used with an indefinite singular, a definite singular, or a bare plural in English. While a kind referring expression such as (1a) predicates a property directly of a kind, a characterizing sentence expresses a regularity about individuals or events, which is made true by having enough members of a kind display the property associated with the kind. In other words, while the predicate meow in (3) can be true of individual members in the cat kind, the property die out expressed in $(1 \mathrm{a} / \mathrm{b})$ can only be true of a kind rather than a single individual.

3. The article system, genericity and kind reference in English, Turkish, Arabic, and Chinese. The languages under investigation in this study are English, Turkish, Arabic, and Chinese. These languages diverge drastically with respect to the article system, presence or absence of plural marking and licensing of bare nominals, which creates an optimum test case to investigate the learnability problem that second language learners face in acquiring subtle meaning distinctions to refer to kinds in a target language. The selection of these particular L1 backgrounds is essential as they are different in terms of the availability of overt determiners. While English has overt indefinite and definite articles, Turkish only has an overt indefinite article but lacks a definite article. Arabic seems to exhibit the reversed pattern compared to Turkish. While it has an overt definite article, it lacks an indefinite article. Chinese, on the other hand, lacks both indefinite and definite articles.

3.1. THE ARTICLE SYSTEM, GENERICITY, AND KIND REFERENCE IN ENGLISH. In English, there are three articles: the definite article the, the indefinite article $a / a n$, and the zero article $\emptyset$. A/an marks indefinite entities, while the marks definite entities. Consider the following example that establishes how an indefinite and a definite article is used.

(4) I saw a cute puppy at the shelter. Then I played with the puppy.

As illustrated above, on the first mention of a cute puppy, there is no presupposition that such a creature exists, which requires the use of the indefinite article $a / n$. Thus, it is infelicitous to use the definite article the. However, on a second mention of an entity, since the presupposition conditions regarding the existence of the entity have been met, a definite article can be used.

As for kind reference in English, it is only possible with a bare plural or a definite singular when count nouns are used. With mass nouns, kind reference is only possible with a bare singular. 


\subsection{THE ARTICLE SYSTEM, GENERICITY, AND KIND REFERENCE IN TURKISH. Although Turkish}

has an indefinite article bir, (a/an or one), it is argued to lack a general article system to overtly mark definiteness or specificity (Lewis, 1967; Underhill, 1976; Erguvanl1, 1984; Kornfilt, 1997; Göksel \& Kerslake, 2005). However, it employs alternative ways such as case morphology, word order, stress, tense, aspect, and modality to encode these functions. The indefinite article bir functions either as an indefinite article or a numeral based on context. Consider the following examples that illustrate this distinction.

(5) hizlı bir araba

fast a car

'a fast car'

(6) bir hizlı araba

one fast car

'one fast car'

As shown above, when a nominal is modified by an adjective + bir (one), bir is interpreted as an indefinite article. However, when bir precedes the adjective, it is interpreted as a numeral. Turkish marks specificity with the accusative marker -(y)I. To refer to a specific entity in the object position, $-(y) I$ is necessary. Consider the minimal pair below.

(7) a. Hakan elma ye-di

Hakan apple eat-PAST.3SG

'Hakan ate an apple / some apples\}.'

b. Hakan elma-y1 ye-di

Hakan apple-ACC eat-PAST.3SG

'Hakan ate a particular apple.'

As shown above, a bare singular noun in object position is compatible with a singular or a plural interpretation. However, it cannot be interpreted as specific without the accusative marker. By contrast, a bare singular or a bare plural noun in subject position in Turkish receive a specific interpretation as shown below.

(8) a. elma masa-da apple table-LOC

'A particular apple is on the table.'

b. elma-lar masa-da apple-PL table-LOC

'The apples are on the table.'

In Turkish, kind reference is established with bare singular (9) or bare plurals (10). Mass nouns are kind referring when bare (11).

(9) sincap Kanada-da yaygın-dir squirrel Canada-LOC common-COP 'Squirrels are common in Canada.'

(10) sincap-lar Kanada-da yaygin-dir squirrel-PLU Canada-LOC common-COP 'Squirrels are common in Canada.' 
(11) süt sağlıkl1-dır

milk healthy-COP

'Milk is healthy.'

3.3. THE ARTICLE SYSTEM, GENERICITY, AND KIND REFERENCE IN ARABIC. Modern Standard Arabic (MSA) has a definite article al- that precedes the noun and does not share gender and number features with the noun (Guella et al., 2008). Despite having received considerable attention in the literature, the question as to whether MSA does indeed have an indefinite article is far from being resolved. There are two opposing views in this realm in the literature. The former postulates an indefiniteness marker attached to the end of the word (Ryding, 2005). The indefinite marker, according to this view takes the form -un for nominative; - an for accusative; and -in for genitive case. According to the second view, MSA has a zero indefinite article to mark indefiniteness (Guella et al., 2008; Sarko, 2008; Lyons, 1999). Consider the two examples with different glosses below, where the former assumes the view that MSA does indeed have an indefinite article, while the latter supports the view that MSA does not have an indefinite article.

(12) MSA with an indefinite article

a. kalb-u-n dog-NOM-IND 'a dog'

b. al-kalb-u the-dog-NOM

'the dog'

c. kelab-u-n dogs-NOM-IND 'dogs'

d. al-kelab-u the-dogs-NOM

'the dogs'

(13) MSA without an indefinite article

a. kalb-un dog-NOM 'dog'

b. al-kalb-u the-dog-NOM 'the dog'

c. kelab-un dogs-NOM 'dogs'

d. al-kelab-u the-dogs-NOM 'the dogs'

Note that the word final suffix or what is known as nunation is usually dropped in MSA and other dialects. Consider the minimal pair from MSA below, where the omission of -un is still acceptable. 
(14) a. hunaaka walad-un ya-drusu fii 1-bayt-i there boy-NOM 3-study in the-house-GEN

'A boy is studying in the house.'

b. hunaaka walad ya-drusu fii 1-bayt-i there boy 3-study in the-house-GEN 'A boy is studying in the house.'

(Aoun et al., 2009, p. 19)

Similarly, -un, considered by some to be the indefinite marker, is dropped in Moroccan Arabic.

(15) Modern Standard Arabic

a. omar muallim-un

omar teacher-NOM

'Omar is a teacher.'

b. al-bayt-u kabir-un

the-house-NOM big-NOM

'The house is big.'

(16) Moroccan Arabic

a. omar muallim

omar teacher

'Omar is a teacher.'

b. d-dar kbira

the-house big

'The house is big.'

(Aoun et al., 2009, p. 35)

The main argument which supports that MSA does not have an indefinite article comes from proper names. Although considered to be individual denoting just like definite descriptions, proper names also receive -un, which casts doubt on the idea that -un is an indefinite article. Based on these points, I argue that MSA lacks an indefinite article.

(17) Aliy-un muallim-un

Ali-NOM teacher-NOM

'Ali is a teacher.'

(Aoun et al., 2009, p. 43)

Kind reference with counts in Arabic is only possible with definite singular (18) or definite plurals (19). Mass nouns also require a definite article for kind reference (20).

(18) al-senjab-u shaeeun fi Kanada

the-squirrel-NOM common in Canada

'Squirrels are common in Canada.'

(19) al-sanajeb-u shaeeatun fi Kanada

the-squirrels-NOM common in Canada

'Squirrels are common in Canada.'

(20) al-haleeb-u sehee

the-milk-NOM healthy

'Milk is healthy.'

3.4. THE ARTICLE SYSTEM, GENERICITY, AND KIND REFERENCE IN CHINESE. Chinese nouns are neither inflected for number nor need a definite or indefinite article. However, the absence of a 
definite and indefinite article does not preclude a bare noun from having a definite, indefinite, or generic interpretation (Huang et al., 2009). Consider the following examples from Huang et al. (2009), where the bare noun gǒu (dog/s) can be interpreted as generic, definite, or indefinite as well as having a singular or a plural interpretation.

(21) 狗 很 聪明

gǒu hen cōngmíng

dog very intelligent

'Dogs are intelligent.'

(22) 我 看到 狗

wǒ kàndào gǒu

I saw dog

'I saw a dog/dogs.'

(23) 狗跑走了

gǒu păo-zǒu-le

dog run-away-le

'The $\operatorname{dog}(\mathrm{s})$ ran away.'

(Huang et al., 2009, p. 283)

In Chinese, a bare singular is used for kind reference for both count, and mass nouns.

(24) 松鼠 在 加拿大很常见

sōngshǔ zài jiānádà hèn chángjiàn

squirrel at Canada very common

'Squirrels are common in Canada.'

(25) 牛奶 很 好喝

niúnăi hěn hăohē

milk very delicious

'Milk is delicious.'

Based on the differences outlined above, we can demonstrate the distribution of articles in kind referring NPs in the four languages ${ }^{1}$.

\begin{tabular}{|l|c|c|c|c|c|}
\hline \multirow{2}{*}{} & \multicolumn{5}{|c|}{ Kind referring NPs } \\
\hline & BS & IS & DS & BP & DP \\
\hline English & & & $\sqrt{ }$ & $\sqrt{ }$ & \\
\hline Turkish & $\sqrt{ }$ & & & $\sqrt{ }$ & \\
\hline Arabic & & & $\sqrt{ }$ & & $\sqrt{ }$ \\
\hline Chinese & $\sqrt{ }$ & & & & \\
\hline
\end{tabular}

Table 1: Crosslinguistic variation in kind referring NPs in the four languages under investigation

4. The current study. I investigated the acquisition of kind referring NPs in L2 English with participants that had Turkish, Arabic, and Chinese L1 backgrounds, comparing their responses to native speakers of English.

${ }^{1}$ BS: bare singular; IS: indefinite singular; DS: definite singular; BP: bare plural; DP: definite plural. 
4.1. RESEARCH QUESTIONS. There are two research questions that I investigate in this study. These are listed below, together with the predictions.

(26) Research questions

a. Is Turkish, Arabic, and Chinese L2 learners' use and understanding of nominals and articles to express kind reference in English affected by the properties, particularly the distribution of articles and number marking of their L1?

Predictions: It is predicted that at least in lower proficiency levels, L2 learners of English will transfer the morphosyntactic manifestation of kind reference interpretation from their L1, making use of articles and plural marking used to mark kind reference in their L1s. It is also predicted that even advanced level L2 learners of English will differ from native speakers in kind reference interpretation since primary linguistic data that the L2 learners are exposed to in English may not be sufficient to guide them to acquire subtle distinctions in meaning to refer to kinds in L2 English.

b. Does L2 proficiency make a difference in L2 learners' correct use and understanding of articles to refer to kinds in English?

Predictions: At higher levels of L2 English proficiency, and with more input and more exposure to the language, L2 learners are expected to behave more native-like in interpreting and producing nominals with kind reference.

4.2. PARTICIPANTS. The L2 learners were all recruited from a large Midwestern university. They were either studying at an intensive English program, or doing their undergraduate or graduate degrees in the US. A total of 37 L2 learners of English with Turkish (10), Arabic (10), and Chinese (10) L1 backgrounds, as well as a native control group (7) participated in the study. The L2 learners were divided into a high proficiency and a low proficiency group based solely on their scores from a 50-item L2 proficiency cloze test (Brown, 1980).

4.3. TASKS. To investigate whether Turkish, Arabic, and Chinese L2 learners of English can successfully acquire kind reference in L2 English, two experimental tasks were employed. These were a Fill in the gaps task (FTG) and an Acceptability judgment task (AJT).

4.3.1. FILL IN THE GAPS TASK. The first task was an untimed Fill in the gaps task (FTG) with 48 items. For each item, participants saw a background sentence, followed by an incomplete sentence and a picture. They were asked to complete the sentences with the clue from the picture. They were also told that they had to use the object in the picture and use any additional words to complete the sentences grammatically. The participants were instructed not to use subject pronouns (it/they), demonstratives (this/that), possessive determiners (his/her/its) or proper names (John/Ashley) so that correct noun phrases with or without articles or plural marking could be elicited. There were 3 experimental conditions, given below followed by an example item that the participants saw in this task.

(27) Experimental conditions in the FTG task ${ }^{2}$

a. Subject/count condition with inherently kind verb or adjectives ( 8 items),

b. Subject/mass condition with inherently kind adjectives (4 items)

c. Object/count condition with inherently kind verbs (4 items).

\footnotetext{
${ }^{2}$ Although the study included those three conditions, only the first one will be reported here due to space limitations. There were 16 more items testing characterizing sentences, and 16 items that were fillers. Thus, there were a total of 48 items in the Fill in the gaps task. These items cannot be discussed here either due to space limitations.
} 
Due to the change in climate and excessive hunting, - may become extinct soon

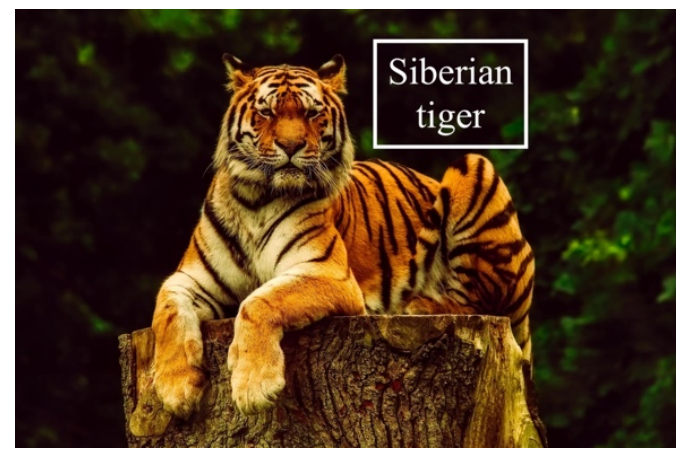

Figure 1: A sample item from the Fill in the gaps task

4.3.2. ACCEPTABILITY JUDGMENT TASK (AJT). Since it is not always possible to elicit the intended forms from participants in an acquisitional study, another task, an Acceptability judgment task (AJT) with 64 items was employed. For each item, participants saw a short sentence with 5 possible continuations, which minimally differed with respect to whether the NP was bare, or if it included plural marking as well as whether there were articles or not. Thus, the first NP in the continuations was always a bare singular noun (BS), followed by an indefinite singular (IS), a definite singular (DS), a bare plural (BP), and a definite plural (DP). On a 4point Likert (1: completely impossible, 2: impossible, 3: possible, 4: perfectly possible) participants were asked to rate each following continuation on how possible they were. They were told that they could give the same rating to different sentences if they thought that they were equally possible or impossible. There were 3 experimental conditions, given below followed by an example item that the participants saw in this task.

(28) Experimental conditions in the $\mathrm{AJT}^{3}$

a. Subject/count condition with inherently kind verb or adjectives (16 items),

b. Subject/mass condition with inherently kind adjectives (8 items),

c. Object/count condition with inherently kind verbs (8 items).

\begin{tabular}{l|l|l|l|l}
$\begin{array}{l}\text { Owing to a decrease in prey base, } \\
\text { hunting, and an encroachment in } \\
\text { habitat by humans, }\end{array}$ & $\begin{array}{l}\text { Completely } \\
\text { impossible }\end{array}$ & Impossible & Possible & $\begin{array}{l}\text { Perfectly } \\
\text { possible }\end{array}$ \\
\hline snow leopard is dying out. & & & & \\
\hline a snow leopard is dying out. & & & & \\
\hline the snow leopard is dying out. & & & & \\
\hline snow leopards are dying out. & & & & \\
\hline the snow leopards are dying out. & & & & \\
\hline
\end{tabular}

Table 2: A sample item from the Acceptability judgment task

4.4. RESULTS. In this results section, the findings based on 2 experimental conditions in 2 different tasks will be presented. These conditions are given below:

(29) a. Fill in the gaps task - Subject/count condition with inherently kind verbs or adjectives

b. AJT - Subject/count condition with inherently kind verb or adjectives

\footnotetext{
${ }^{3}$ Although the study included those three conditions, only the first one will be reported here due to space limitations. There were 32 more items testing characterizing sentences in the AJT. Therefore, there were a total of 64 items. These items, however, cannot be discussed here either due to space limitations.
} 
For each of the experimental conditions, a separate generalized linear mixed effects model was run in R, using the lmer4 function. The fixed effects were (a) L1 (Turkish, Arabic, Chinese, versus native English speakers), (b) level (high and low for the L2 group), and (c) type of response (BS: bare singular, IS: indefinite singular, DS: definite singular, BP: bare plural, and DP: definite plural). The main effects included L1, level, and type. Interaction effects included: L1*level, L1*type, level*type, and L1*level*type. Finally, random effects were the participants and the items. The responses of the NS group were compared to the L2 groups, and the responses of L2 groups were also compared to each other. Significant interactions were followed up with post-hoc comparisons. These pairwise comparisons were conducted using the emmeans function in R using Tukey for adjustments for multiple comparisons.

The experimental condition in the Fill in the gaps task that is of interest in this study is the subject/count condition with inherently kind verb or adjectives. This condition tests whether the participants were able to provide the correct response, which is a definite singular or a bare plural, for the gaps in sentences with kind referring NPs. The responses that the participants provided for each blank was categorized as belonging to one of five possible NP categories. These were: a bare singular (BS), an indefinite singular (IS), a definite singular (DS), a bare plural (BP), and a definite plural (DP). The table below illustrates participants' responses as average percentages for each item type.

\begin{tabular}{lccccc} 
& BS & IS & DS & BP & DP \\
\hline Arabic low & 33.33 & 8.33 & 27.22 & 30.55 & 0 \\
\hline Arabic high & 16.66 & 2.08 & 25 & 56.25 & 0 \\
\hline Chinese low & 25 & 0 & 18.05 & 56.94 & 0 \\
\hline Chinese high & 29.8 & 0 & 12.5 & 56.73 & 0.96 \\
\hline Turkish low & 12.5 & 4.54 & 3.4 & 79.54 & 0 \\
\hline Turkish high & 7.95 & 0 & 27.27 & 57.95 & 6.81 \\
\hline NS & 0.52 & 0 & 46.35 & 53.12 & 0 \\
\hline
\end{tabular}

Table 3: Participants' suppliance of different NP forms in the subject/count condition in the Fill in the gaps task

The results indicated a main effect of item type $(\mathrm{p}=0.002)$, and interaction effects of level*type $(p=0.002)$, and $\mathrm{L} 1 *$ type $(p=0.001)$. As the level did not create a significant difference within the L2 learners, the significant results are the post-hoc comparisons on the interaction between L1 and type. In this domain, Arabic learners differed from NS in that they provided bare singulars significantly more $(p=0.024)$. Similarly, Chinese L2 learners provided significantly more bare singulars than the NS group in this experimental condition as well (Chinese - English $(p=0.003)$ ). Interestingly, Turkish participants, whose L1 licenses a bare singular for kind reference, was not significantly different from the NS group in this condition. Another NP type in which significant differences were observed was the definite singulars. Both Chinese and Turkish L2 learners of English differed from the NS group in that they provided significantly less definite singulars in the subject count condition with inherently kind adjective or verbs $(p=0.003)$. Arabic L2 learners of English, whose L1 licenses a definite singular for kind reference, was not significantly different from the native speaker group in this domain.

The second experimental task was an Acceptability Judgment Task (AJT). The AJT tests participants' judgments in terms of how possible or impossible they think the 5 NP forms were following a short introductory sentence. They rated those sentences on a Likert scale from 1 to 4 . 
The ratings that the participants gave for each NP form was averaged for each participant, and the average rating for each experimental condition was calculated.

The experimental condition in the AJT that is of interest in this study is the subject/count condition with inherently kind verb or adjectives. The correct responses, and the ones that are expected to receive high acceptability ratings in this condition were the definite singular and a bare plural. The table below illustrates participants' ratings in averages for each NP type.

\begin{tabular}{lccccc} 
& BS & IS & DS & BP & DP \\
\hline Arabic low & 2.30 & 2.05 & 2.90 & 2.93 & 3.16 \\
\hline Arabic high & 2.11 & 2.25 & 3.47 & 3.87 & 3.48 \\
\hline Chinese low & 2.41 & 1.61 & 2.88 & 3.25 & 2.54 \\
\hline Chinese high & 2.67 & 1.41 & 2.97 & 3.88 & 2.79 \\
\hline Turkish low & 1.86 & 1.94 & 2.25 & 3.41 & 2.37 \\
\hline Turkish high & 1.86 & 1.25 & 2.92 & 3.89 & 2.45 \\
\hline NS & 1.19 & 1.10 & 3.85 & 3.98 & 2.28 \\
\hline
\end{tabular}

Table 4: Participants' ratings of different NP forms in the subject/count condition in the Acceptability judgment task

The results from the generalized linear mixed effects model run in $\mathrm{R}$ indicated a main effect of level $(p=0.001), \mathrm{L} 1(p=0.002)$, and NP type $(p=0.000)$. There were also significant interaction effects of level*type $(p=0.000)$, and L1*type $(p=0.002)$. In terms of level*type $(p=0.000)$ interaction, the low L2 proficiency groups rated indefinite singulars significantly higher than high proficiency L2 groups ( $p=0.002$ ). Another significant difference between the low and high L2 proficiency groups was that the former group rated the definite singulars lower than the latter $(p=0.023)$.

As for L1*type ( $p=0.001)$ interaction, there were significant differences between the L2 learner groups and the NS groups in all 5 NP types. First of all, both Arabic and Chinese L2 learners of English differed from the NS in that they rated bare singulars higher (Arabic English $(p=0.005)$; Chinese - English $(p=0.000)$ ).

In indefinite singular NP type, the Arabic participants differed from all the other groups including the NS group as they rated indefinite singulars significantly higher than other groups (Arabic - English $(p=0.000)$; Arabic - Chinese $(p=0.009)$; Arabic - Turkish $(p=0.033)$ ). In this NP type, there was also a significant difference between Turkish participants and the NS group since Turkish L2 learners of English rated indefinite singulars higher than the NS group $(p=0.026)$.

In definite singular NP type, the NS group differed from all 3 L2 learner groups in giving higher ratings to definite singulars in the subject/count condition with inherently kind verb and adjectives. The difference for all three comparisons was significant (Arabic - English $(p=0.044)$, Chinese - English $(p=0.000)$; Turkish - English $(p=0.000)$ ).

Regarding bare plurals as kind referring NPs, the only significant difference was between the Arabic participants and the NS group. The Arabic L2 learners of English rated bare plurals significantly lower than the NS group $(p=0.017)$.

Finally, Arabic participants rated definite plurals significantly higher than the NS group $(p=0.003)$, as well as the Turkish participants $(p=0.014)$.

5. Conclusion. The results of this study indicated that; (a) Turkish, Arabic, and Chinese L2 learners' use and understanding of nominals and articles to express kind reference in English was 
indeed affected by the properties, particularly the distribution of articles and number marking of their L1; (b) L2 proficiency made a slight but generally insignificant difference in L2 learners' correct use and understanding of articles to refer to kinds in English; and (c) even at higher proficiency levels, L2 learners' use and understanding of nominals and articles to express kind reference in English was not native-like.

When we scrutinize the responses of the native speakers in the subject/count condition with inherently kind verb and adjectives in the Fill in the gaps task, we can see that the NP forms that they provide in this condition are quite expected. They consistently provided definite singulars (46.35 percent) or bare plurals (53.12 percent) for kind reference. The native speakers produced bare plurals significantly more for kind reference. These results are crucial because they provide the baseline against which L2 learners' responses can be evaluated. The native speakers judged all other NP forms as impossible to refer to kinds.

As for L2 learners, there is a great deal of variation in the NP forms they produced for kind reference. We see that Arabic learners at low proficiency levels mainly oscillated between bare singulars (33.33 percent), definite singulars (27.77 percent), and bare plurals (30.55 percent), only the last two of which are allowed as kind reference in English. Arabic participants at higher proficiency levels produced bare plurals significantly more than other NP forms while still accepting bare singulars at 16.66 percent. In the Chinese groups, we see almost identical results. Bare plurals made up about 60 percent of the responses, followed by definite singulars (around 30 percent), and bare singulars (around 15 percent). A major observation about the Turkish group is that at lower levels, they mainly produced bare plurals for kind reference. However, in the high proficiency group, bare plurals were produced significantly less than the low proficiency group. What is more, Turkish participants in the high proficiency group produced definite singulars significantly more than the participants in the low proficiency group.

There are two points worth mentioning in the results that demonstrate L1 transfer. The first piece of evidence is the production of definite singulars for kind reference at around 30 percent for both the low and high proficiency Arabic group. As explained before, Arabic uses definite singulars for kind reference. We see that even in lower proficiency levels, Arabic learners did produce definite singulars significantly more than the high and low Chinese groups, as well as the low Turkish group, whose L1s lack a definite singular. The only counterexample is the high proficiency level Turkish group, who also produced definite singulars at about 30 percent.

The second piece of information that potentially points to L1 transfer is the persistence of bare singulars in both the high and the low Chinese groups, who produced bare singulars for kind reference at about 30 percent. As stated previously, Chinese allows bare singulars for kind reference, which is why these results are compatible with L1 transfer.

Despite the two points above that indicate potential L1 transfer, there are two findings that are unexpected if $\mathrm{L} 1$ transfer is at play. The first is relatively higher production of bare singulars by the low and high proficiency Arabic group (33.33 and 16.66 percent respectively). Arabic disallows bare singulars in every context. Thus, if $\mathrm{L} 1$ transfer is postulated, these findings are puzzling. I believe that the Arabic learners may have just avoided more difficult NP forms and preferred the bare singular as a default strategy especially in the lower proficiency group. Although the bare singular for count nouns is barely found in the English input, Arabic learners may have simply provided the bare singular as an avoidance strategy since it is much easier to provide a bare singular than to correctly control the morphosyntax of a given NP in a certain context.

Yet another point that is perplexing if L1 transfer is at play is Turkish L2 learners' relatively low production of bare singulars in both proficiency groups although bare singulars 
can be kind referring in Turkish. In both the Arabic and the Turkish group, however, bare singulars were produced less as the proficiency in the L2 went up, which still demonstrates that L2 proficiency or the primary linguistic data (PLD) was guiding L2 learners to be more nativelike despite not creating a significant difference within the L1 groups.

The Acceptability judgment task also provides some insight into the interlanguage representation and development of kind reference in L2 English as well as demonstrating some interesting patterns from native speakers. I want to emphasize the findings from the native speaker group since there is a pattern that was not observed in the Fill in the gaps task. First of all, in the subject/count condition with inherently kind verb or adjectives, the native speakers judged both bare plurals and definite singulars as perfectly possible for kind reference, while rating bare singulars and indefinite singulars as completely impossible. However, definite plurals, which the native speakers did not produce at all in the Fill in the gaps task, were not as straightforward for native speakers. They judged those as impossible for kind reference, but they did not reject them as strongly as they did bare singulars and indefinite singulars.

As for L2 learners, their judgments were not as straightforward as the native speakers. All the L2 learner groups, with the exception of the Arabic learners at low proficiency levels, rated bare plurals higher than other NP types. First of all, the Arabic learners at both proficiency levels shunned rating any type of NP as completely impossible. Thus, their average ratings ranged from 2.05 to 3.87. The Arabic learners at low proficiency levels rated definite singulars, bare plurals, and definite plurals as possible for kind reference. Their ratings for those three NP types increased in the high proficiency group. Strikingly, we see that the Arabic group rated definite plurals significantly higher than the other learner groups. We can attribute this to the fact that definite plurals can be kind referring in Arabic. On the other hand, both Chinese groups rated bare singulars higher than the other two learner groups, with the difference reaching significant levels when compared to the Turkish group. This finding is interesting because both Turkish and Chinese allow bare singulars for kind reference. However, the Chinese group rated bare singulars significantly higher. One last interesting finding is that definite plurals are the third most highly rated NP type among the learner groups.

6. Future directions. As explained before, the languages under investigation in this study make use of different morphosyntax for kind reference. This brings up the question of whether crosslinguistic variation is due to the lexical items or to the functional projections (DP/NumP) above the NP, particularly to the information encoded in the article system. I believe that a feature based account may give us a solid perspective on the source of kind reference crosslinguistically. Developing a feature based account for kind reference broadly requires answers to three separate questions/matters:

(30) Future directions in investigating genericity and kind reference

a. Is there a feature responsible for kind reference? If yes, what exactly is that feature? How do we know that that feature is a primitive feature in language and human cognition?

b. Where is the feature? Is it on the nominal, or encoded on a functional projection such as the Determiner Phrase (DP), Classifier Phrase (ClP), or Numeral Phrase (NumP)?

c. Since not all languages have overt determiners, if we postulate that kind reference feature is encoded on a functional projection above the NP in a language where kind reference can be established with a bare nominal, then it also requires independent evidence to show that nouns are not so bare in that language (such as in Turkish and Chinese). 
Were we to postulate features within the nominal domain that lead to kind reference, some possible candidates for those features would be [+generic], [+kind referring], [+taxonomic], or finally a negative feature [-referential]. The only authors that discussed one of these features is Ionin et al. (2011). They maintain that in addition to encoding definiteness, the definite article in English encodes the feature [+taxonomic]. However, they do not speculate on the conditions under which this feature is encoded on the definite article. That is, the definite article the cannot simultaneously encode the features [+definite] and [+taxonomic] since that would lead to a crash in interpretation as an NP cannot be referential [ + definite], and non-referential $[+$ taxonomic] at the same time.

Another issue relates to the syntactic position of the feature. That is, whether it is on the nominal, or encoded on a functional projection such as the Determiner Phrase (DP), Classifier Phrase (CIP), or Numeral Phrase (NumP). I believe that crosslinguistic variation in the denotation of nominals stems from the functional projections above the NP, and particularly features encoded on them. Future studies on genericity and kind reference should investigate those questions.

\section{References}

Aoun, Joseph E, Elabbas Benmamoun \& Lina Choueiri. 2009. The syntax of Arabic. Cambridge: Cambridge University Press.

Carlson, Gregory N. \& Francis Jeffrey Pelletier. 1995. The generic book. Chicago: University of Chicago Press.

Erguvanl, Eser Emine. 1984. The function of word order in Turkish grammar, Vol. 106. Berkeley: University of California Press.

Göksel, Aslı \& Celia Kerslake. 2005. Turkish: A comprehensive grammar. New York: Routledge.

Guella, Hakima, Viviane Deprez \& Petra Sleeman. 2008. Article choice parameters in L2. Proceedings of the Generative Approaches to Second Language Acquisition Conference 9. 57-69.

Huang, Cheng-Teh James, Yen-hui Audrey Li \& Yafei Li. 2009. The syntax of Chinese. Cambridge: Cambridge University Press.

Ionin, Tania \& Silvina Montrul. 2010. The role of L1 transfer in the interpretation of articles with definite plurals in L2 English. Language Learning 60(4). 877-925.

Ionin, Tania, Silvina Montrul, Ji-Hye Kim \& Vadim Philippov. 2011. Genericity distinctions and the interpretation of determiners in second language acquisition. Language Acquisition, 18(4). 242-280. https://doi.org/10.1111/j.1467-9922.2010.00577.x.

Ionin, Tania, Elaine Grolla, Silvina Montrul \& Hélade Santos. 2014. When articles have different meanings: Acquiring the expression of genericity in English and Brazilian Portuguese. In Anne Zribi-Hertz \& Patricia Cabredo Hofher (eds.), Crosslinguistic studies on noun phrase structure and reference. 367-397. Leiden: Brill Academic Publishers.

Kornfilt, Jaklin. 1997. Turkish. Routledge.

Krifka, Manfred, Francis Jeffrey Pelletier, Gregory N Carlson, Gennaro Chierchia, Godehard Link \& Alice Ter Meulen. 1995. Genericity: An introdcution. In Gregory N. Carlson \& Francis Jeffrey Pelletier (eds.), The generic book. 1-124. Chicago: Chicago University Press.

Lewis, Geoffrey. 1967. Turkish grammar. Oxford: Clarendon.

Lyons, Christopher. 1999. Definiteness. Cambridge: Cambridge University Press.

Ryding, Karin C. 2005. A reference grammar of modern standard Arabic. Cambridge: Cambridge University Press.

Sarko, Ghisseh. 2008. Morphophonological or syntactic transfer in the acquisition of English articles by L1 speakers of Syrian Arabic. Proceedings of the Generative 
Approaches to Second Language Acquisition Conference 19.

Schwartz, Bonnie D. \& Rex A. Sprouse. 1996. L2 cognitive states and the full transfer/full access model. Second Language Research 12(1). 40-72.

Underhill, Robert. 1976. Turkish grammar. Cambridge, MA: MIT Press. 